\title{
Autologous graft versus myeloma: it's not a myth
}

\author{
Shuai Dong and Irene M. Ghobrial
}

Department of Medical Oncology, Dana-Farber Cancer Institute, Harvard Medical School, Boston, Massachusetts, USA.

\begin{abstract}
Graft-versus-tumor (CVT) effects have been thought to mostly result from allogeneic transplants; however, there is a growing body of research that supports a possible autologous CVT effect. In early clinical studies, a positive correlation between lymphocyte count recovery after autologous transplantation and overall survival has been observed. However, mechanistic studies to identify the mediators of autologous CVT responses have been lacking. In this issue of the $J C I$, Vuckovic et al. observed a T celldependent autologous CVT effect in the Vk* MYC myeloma model. Moreover, the authors showed that CD8 $\mathrm{T}$ cells mediate myeloma control through IFN- $\gamma$ secretion, which could be further augmented with a CD137 agonist, suggesting a therapeutic approach for enhancing autologous GVT.
\end{abstract}

\section{Current approaches for multiple myeloma}

Multiple myeloma (MM), characterized by the accumulation of malignant antibody-producing plasma cells, is the second most common blood cancer after non-Hodgkin lymphoma (NHL) in the United States (1). Thanks to the understanding of disease biology, an increasing number of individualized treatments are available that have largely improved patient outcomes. Despite the large number of experimental therapeutics being tested for myeloma, such as immunotherapies, histone deacetylase (HDAC) inhibitors, chimeric antigen receptor (CAR) $\mathrm{T}$ cell therapy, MM remains incurable. For transplant-eligible patients, high-dose therapy (HDT) followed by autologous stem cell transplantation (SCT) is the standard of care (1). While allogeneic transplantation can provide a potential cure due to the graft-versus-tumor (GVT) effect, this approach is prohibitive because of the high risk of treatment-associated mortality, including the development of graft-versus-host disease (GVHD). Especially for patients with $\mathrm{MM}$, allogeneic SCT does not confer superior overall survival (OS) compared with autologous SCT. Therefore, allogeneic SCT is not considered a first-line treatment for MM and is only done in clinical trial settings. On the other hand, post-autologous transplant patients who received maintenance therapy with the immunomodulatory (IMiD) agent lenalidomide had remarkably improved survival rates compared with those who did not, suggesting that the host's immune system can be improved to keep the cancer at bay (2).

There is a significant body of evidence suggesting that the immune system has a critical role in myeloma disease control. IMiDs have been shown to improve the function of NK cells and $\mathrm{T}$ cells, both of which contribute to disease regression $(3,4)$. With the immunomodulatory effects of IMiDs, it remains elusive whether patients are capable of generating spontaneous antitumor immunity. An array of early clinical stud-

\section{Delated Article: p. 106}

Conflict of interest: IMG has a consulting/advisory role with Celgene, Takeda, Bristol-Myers Squibb, Janssen Pharmaceuticals, and Amgen. IMG has also received research funding and honoraria from Celgene, Takeda, Bristol-Myers Squibb, Janssen Pharmaceuticals, and Amgen.

Reference information: / Clin Invest. 2019;129(1):48-50. https://doi.org/10.1172/JCI125431. ies have reported a positive correlation between lymphocyte count recovery after autologous SCT and OS, suggesting an autologous GVT effect. Many of these studies were done retrospectively and involved a wide range of diseases, including MM, B cell/T cell NHL, Hodgkin lymphoma (HL), mantle cell lymphoma, acute myeloid leukemia, metastatic breast cancer, and ovarian cancer (5). These studies found that, among the autograft immune effector cells, $\mathrm{CD} 4^{+} \mathrm{T}$ cells (in $\mathrm{MM}$ ), NK cells (in diffuse large B cell lymphoma), and cytotoxic DCs (in classical HL) are associated with better clinical outcomes. For post-autologous SCT MM patients, it was reported that a higher $\mathrm{CD}^{+} \mathrm{T}$ cell count and an increase in the ratio of $\mathrm{CD}^{+}{ }^{+}$to $\mathrm{CD} 8^{+} \mathrm{T}$ cells were associated with superior outcomes (6). However, mechanistic studies on the factors that mediate autologous GVT have been lacking.

\section{Mechanisms of autologous GVT in MM}

In this issue, Vuckovic et al. used the $\mathrm{Vk}^{*}$ MYC murine myeloma model to examine spontaneous $\mathrm{T}$ cell antimyeloma immunity in the autologous transplant setting (7). Bone marrow transplantation (BMT) with myeloma-experienced BM performed in myeloma-bearing mice induced clonespecific, T cell-dependent disease control. This effect was generated by memory $\mathrm{T}$ cells within the myeloma-experienced graft as well as by priming of naive T cells. Compared with the mice that received myeloma-free $\mathrm{BM}, \mathrm{CD}^{+} \mathrm{T}$ cells from mice that received myeloma-experienced $\mathrm{BM}$ had distinct $\mathrm{T}$ cell receptor (TCR) repertoires and greater clonotype overlap. These $\mathrm{CD}^{+} \mathrm{T}$ cells induced antimyeloma immunity through IFN- $\gamma$ secretion, which could be further enhanced by a CD137 (also known as 4-1BB) agonist and PD-1 blockade. Furthermore, Vuckovic and colleagues showed that secretion of IL-17A from donor BM directly stimulated myeloma cells through the IL-17 receptor 


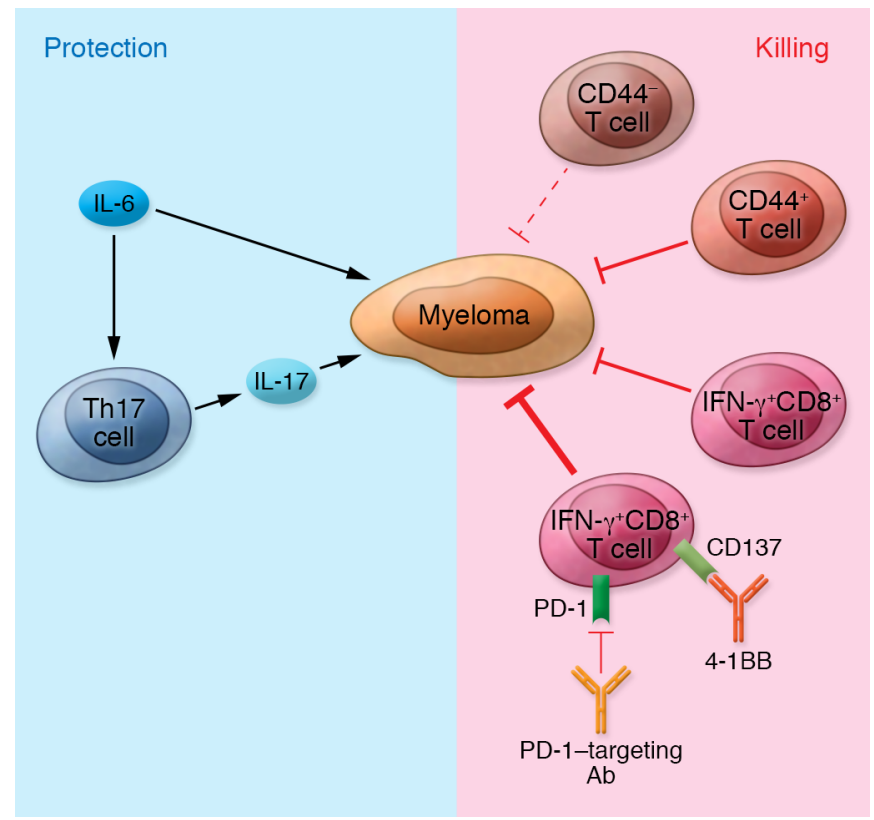

to promote tumor growth and immune evasion, which was independent of Th17 cell differentiation (Figure 1).

In contrast to clinical observations, Vuckovic et al. found that $\mathrm{CD}^{+} \mathrm{T}$ cells, rather than $\mathrm{CD} 4^{+} \mathrm{T}$ cells or NK cells, are critical for autologous GVT (7). This observation raises the question as to whether this discrepancy is due to the murine model and/or the tumor clone used. Could this $\mathrm{CD}^{+} \mathrm{T}$ cell dependency be a specific phenomenon for the $\mathrm{Vk}^{*} \mathrm{MYC}$ model? Is it possible that specific antigens with this clone triggered a $\mathrm{CD}^{+} \mathrm{T}$ cell response? The authors emphasized that the observed antimyeloma immunity was clone specific. Had the T cells experienced a different myeloma cell clone, would the observed outcome be different? It will be worthwhile to confirm this observation in a different murine myeloma model. In addition, the authors examined the TCR $\beta$ chain diversity and clonal types. It is likely that this particular TCR $\beta$ chain profile reflects the myeloma cell clone used in the study. Is TCR $\beta$ clonality the key for the autologous GVT effect? Given the heterogeneity of human disease, are similar profiles observed in patients? What are the factors influencing the clonality? How can we incorporate this information into predicting disease control or relapse?

Vuckovic and colleagues also reported that the most effective antimyeloma $\mathrm{CD}^{+}$ $\mathrm{T}$ cells were within the $\mathrm{CD} 44^{+}$subset, sug- gesting that antigen experience contributes to antitumor immunity (7). Mice that received myeloma-experienced $\mathrm{CD} 44^{+} \mathrm{T}$ cells were found to have an expansion of $\mathrm{CD} 8^{+} \mathrm{T}$ cells but not $\mathrm{CD} 4^{+} \mathrm{T}$ cells. It is not surprising that both activated DNAM- $1^{+}$ PD $-1^{+} \mathrm{CD}^{+} \mathrm{T}$ cells as well as exhausted DNAM-1-PD-1+Tim- $3^{+} \mathrm{CD}^{+} \mathrm{T}$ cells were observed upon engraftment of $\mathrm{CD} 44^{+} \mathrm{T}$ cells. Nonetheless, it is impressive to see that, despite having an exhausted phenotype, these $\mathrm{CD} 44^{+} \mathrm{T}$ cells still exhibited superior antimyeloma immunity. Guillerey et al. recently reported that the exhaustion marker TIGIT was upregulated on $\mathrm{CD}^{+} \mathrm{T}$ cells during disease progression (8). This TIGIT expression was also the reason for disease relapse in autologous transplantation in the $\mathrm{Vk}^{*} \mathrm{MYC}$ mouse (9). However, Vuckovic and colleagues did not mention TIGIT in this study. Is TIGIT also expressed in the myeloma-experienced graft? What could have contributed to the differences in disease control? Paradoxically, it has been reported that human effector $\mathrm{CD}^{+} \mathrm{T}$ cells derived from naive cell subsets possess better quality for adoptive immunotherapy compared with those derived from memory cell subsets (10). Perhaps the transgenic expression of antigen receptor and forced expansion "aged" the memory $\mathrm{T}$ cells differently in the human setting. Nonetheless, it will be extremely intriguing to see whether the myelomaexperienced $\mathrm{T}$ cells from human patients also exhibit superior antimyeloma immunity.
Figure 1. Autologous GVT effects are mediated by IFN- $\gamma$-expressing $\mathrm{CD8}^{+} \mathrm{T}$ cells. Expansion of CD8 ${ }^{+} T$ cells from autologous, myeloma-experienced $\mathrm{BM}$ promotes antimyeloma effects. Of the transplanted $\mathrm{CD}^{+} \mathrm{T}$ cell population, $\mathrm{CD} 44^{+}$ cells are the most effective at killing myeloma cells, suggesting that antigen experience is critical for antitumor immunity. Th17 cells support myeloma cells via IL-17A secretion, though this effect is not the result of enhanced Th17 cell differentiation. The antimyeloma effects of $\mathrm{CD} 8^{+} \mathrm{T}$ cells are mediated by the secretion of IFN- $\gamma$, and this immunity can be enhanced by treatment with a CD137 agonist and PD-1 blockade.

Since Th17 cells have been suggested to induce myeloma progression through IL-17 production (11), Vuckovic and colleagues undertook the task to elucidate the role of IL-17 after autologous transplantation. In this setting, myeloma development was impaired following IL-17 neutralization, confirming the negative impact of IL-17 on disease control (7). The effect of IL-17 is probably not due to its action on the donor graft, as the study by Vuckovic et al. showed that transplantation with IL-17 receptor-deficient donor BM did not notably reduce disease burden. When both donor and recipient BM were lacking the IL-17 receptor, there was a systemic increase in IL-17 levels, which further induced the activation of multiple pathways involved in myeloma survival and immune evasion. However, the study did not rule out the possible effect of IL-17 on the recipient's BM compartment.

IFN- $\gamma$ is thought to be an integral part of antitumor immunity. However, depending on the context, it can either be immune activating or immune suppressing. IFN- $\gamma$ can promote antitumor immunity through direct cytotoxic effects on tumor cells, activation of macrophages, and inhibition of FoxP3 ${ }^{+}$Treg function $(12,13)$. Vuckovic and colleagues showed that IFN- $\gamma$ is critical for the $\mathrm{CD}^{+} \mathrm{T}$ cells to control myeloma. On the other hand, IFN- $\gamma$ has also been shown to induce PD-L1 expression on ovarian cancer cells, leading to immune eva- 
sion (14). Additionally, our group recently observed that myeloma cells can produce IFN- $\gamma$, which leads to Treg expansion (15). These data again highlight the dual effects of IFN- $\gamma$ in myeloma-immune microenvironment crosstalk. Nonetheless, Vuckovic et al. demonstrated that IFN- $\gamma$-producing, $\mathrm{CD}^{+} \mathrm{T}$ cell-dependent antimyeloma immunity can be further enhanced by treatment with a CD137 agonist. The agonist produced activation and exhaustion profiles, including PD-1 upregulation, similar to those seen in mice transplanted with myeloma-experienced BM. Moreover, the addition of PD-1 blockade with the CD137 agonist further improved $\mathrm{T}$ cell-mediated myeloma control.

\section{Concluding remarks}

In summary, these data provide important insight into spontaneous host antimyeloma immunity and the crosstalk between myeloma cells and the T cell compartment. Although it remains to be determined whether this phenomenon can be translated to the human setting, this study suggests a possible new targeting strategy to improve post-transplantation recovery and perhaps clear minimal residual disease. Furthermore, it will be intriguing to examine whether the autologous GVT effect is myeloma specific or can also be found in other hematological malignancies.

\section{Acknowledgments}

We acknowledge Mark Bustoros (DanaFarber Cancer Institute) for helpful comments and suggestions regarding this Commentary.

Address correspondence to: Irene M. Ghobrial, Harvard Institute of Medicine Room 240, 4 Blackfan Circle, Boston, Massachusetts 02115, USA. Phone: 617.632.6777; Email: Irene_Ghobrial@DFCI.harvard.edu.

1. Kumar SK, et al. Multiple myeloma. Nat Rev Dis Primers. 2017;3:17046.

2. Roeven MW, Hobo W, Schaap N, Dolstra $\mathrm{H}$. Immunotherapeutic approaches to treat multiple myeloma. Hum Vaccin Immunother. 2014;10(4):896-910.

3. Haslett PA, Corral LG, Albert M, Kaplan G. Thalidomide costimulates primary human T lymphocytes, preferentially inducing proliferation, cytokine production, and cytotoxic responses in the CD8 ${ }^{+}$subset. J Exp Med. 1998;187(11):1885-1892.

4. Reddy N, et al. Immunomodulatory drugs stimulate natural killer-cell function, alter cytokine production by dendritic cells, and inhibit angiogenesis enhancing the anti-tumour activity of rituximab in vivo. Br J Haematol. 2008;140(1):36-45.

5. Porrata LF. Autologous graft-versus-tumor effect: reality or fiction? Adv Hematol. 2016;2016:5385972.
6. Schmidmaier R, Oversohl N, Schnabel B, Straka C, Emmerich B. Helper T cells $\left(\mathrm{CD}^{+} / \mathrm{CD}^{+}\right)$ within the autologous peripheral blood stem cell graft positively correlate with event free survival of multiple myeloma patients. Exp Oncol. 2008;30(3):240-243.

7. Vuckovic S, et al. Bone marrow transplantation generates T cell-dependent control of myeloma in mice. JClin Invest. 2019;129(1):106-121.

8. Guillerey C, et al. TIGIT immune checkpoint blockade restores $\mathrm{CD} 8^{+} \mathrm{T}$-cell immunity against multiple myeloma. Blood. 2018;132(16):1689-1694.

9. Minnie SA, et al. Myeloma escape after stem cell transplantation is a consequence of T-cell exhaustion and is prevented by TIGIT blockade. Blood. 2018;132(16):1675-1688.

10. Hinrichs CS, et al. Human effector $\mathrm{CD}^{+} \mathrm{T}$ cells derived from naive rather than memory subsets possess superior traits for adoptive immunotherapy. Blood. 2011;117(3):808-814.

11. Prabhala RH, et al. Elevated IL-17 produced by TH17 cells promotes myeloma cell growth and inhibits immune function in multiple myeloma. Blood. 2010;115(26):5385-5392.

12. Mosser DM, Edwards JP. Exploring the full spectrum of macrophage activation. Nat Rev Immunol. 2008;8(12):958-969.

13. Overacre-Delgoffe AE, et al. Interferon- $\gamma$ drives treg fragility to promote anti-tumor immunity. Cell. 2017;169(6):1130-1141.e11.

14. Abiko K, et al. PD-L1 on tumor cells is induced in ascites and promotes peritoneal dissemination of ovarian cancer through CTL dysfunction. Clin Cancer Res. 2013;19(6):1363-1374.

15. Kawano Y, et al. Blocking IFNAR1 inhibits multiple myeloma-driven Treg expansion and immunosuppression. JClin Invest. 2018;128(6):2487-2499. 